\title{
A wandering path toward prevention for acute kidney injury
}

\author{
Simon J. Atkinson \\ Department of Biology, Indiana University-Purdue University Indianapolis, Indianapolis, Indiana, USA
}

\begin{abstract}
Acute kidney injury (AKI) is a common cause of hospital-related mortality; therefore, strategies to either prevent or treat this complication are of great interest. In this issue of the $J C I$, Inoue, Abe, and colleagues have uncovered a targetable neuroimmunomodulatory mechanism that protects mice from ischemia-reperfusion injury (IRI) and subsequent AKI. Specifically, the authors demonstrate that vagus nerve stimulation (VNS) activates the cholinergic antiinflammatory pathway (CAP), resulting in activation of antiinflammatory effects via $\alpha 7$ nicotinic acetylcholine receptor-expressing splenic macrophages. Together, the results of this study have potential clinical implications in the prevention of AKI in at-risk individuals.
\end{abstract}

that ultrasound stimulation might invoke an antiinflammatory response, leading Okusa and colleagues to propose that this protective effect was due to the known ability of certain ultrasound frequencies to stimulate nerves. The proposed mechanism of action (Figure 1) involves ultrasound activation of adrenergic neurons innervating the spleen, stimulation of $\mathrm{CD}^{+}$cells via $\beta$-adrenergic receptors, consequent release of acetylcholine by $\mathrm{T}$ cells, and then stimulation of nicotinic cholinergic receptors on myeloid/macrophage cells. Pharmacologic studies have implicated stimulation of $\alpha 7$ nicotinic acetylcholine receptors ( $\alpha 7 \mathrm{nAChRs})$ present on the myeloid cells in promoting an antiinflammatory response by these cells (7). Subsequent work from the Okusa lab (8) showed that the protective effect of ultrasound did indeed require innervation from the splenic nerve and was accompanied by attenuation of circulating and renal IL- 6 in the setting of IRI. Moreover, the beneficial effect of ultrasound stimulation on myeloid cells in treated animals was persistent over several days and sufficiently durable to convey protection from IRI to ultrasound-naive animals that had received myeloid cells from ultrasoundstimulated animals. The protective effect of ultrasound also decreased severe sepsisinduced AKI in a mouse model. This latter observation of protection in a distinct (and clinically important) kidney injury model is particularly encouraging, as many interventions that have been shown to be effective in a single rodent AKI model have not proved to be clinically useful. Overall, these studies established the previously described cholinergic antiinflammatory pathway (CAP) (9) as the central mechanism of protection conferred by ultrasound stimulation.

\section{Vagus nerve stimulation-} mediated protection from IRI

While these observations are promising, questions remain as to how best to both 


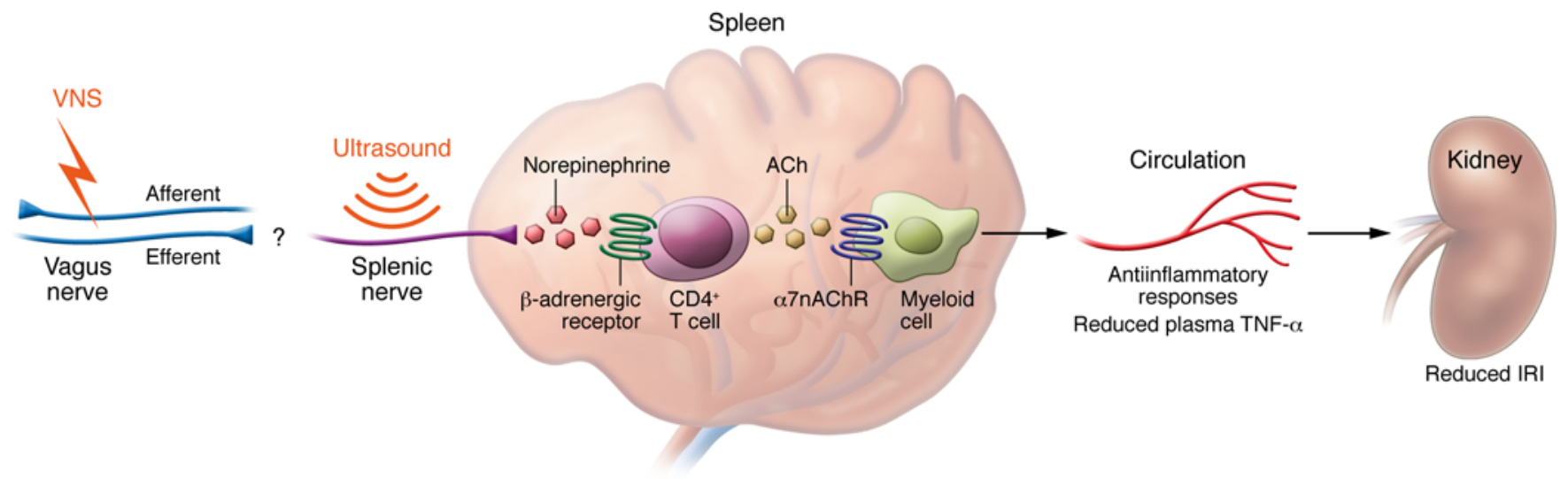

Figure 1. Activation of the CAP by ultrasound or VNS attenuates IRI. Both VNS and ultrasound prior to IRI in murine models protect against the development of AKI. This protective effect is mediated by the integration of neural signals and $\alpha 7 n A C h R$ on splenic macrophages. In addition to reducing AKI, VNS also reduced plasma levels of the proinflammatory cytokine TNF- $\alpha$. Adapted with permission from the Journal of the American Society of Nephrology (6).

extend these findings to a therapeutic intervention that could be implemented in clinical practice and to uncover the precise neural mechanisms involved. Vagus nerve stimulation (VNS), in which a pattern of electrical stimulation is delivered to the vagus nerve from a pulse generator implanted in the chest (with noninvasive transcutaneous devices in development), is approved for treatment of epilepsy and depression (10). Studies in animal models to evaluate the use of VNS in brain and heart IRI, for example, suggest that this strategy is capable of activating the CAP reflex (7). In this issue, Inoue, Abe, and colleagues (5) tested to determine whether VNS could produce protective effects similar to those induced by ultrasound stimulation of the spleen. The results of this study confirm that VNS ameliorates IRI in the kidney and that the effect depends on the same CAP reflex pathway that is activated by ultrasound.

An interesting finding of the study by Inoue, Abe, et al. was that stimulation of either vagal afferents or efferents is sufficient to confer protection (5). Additionally, activation of vagal afferents on the left-hand side stimulated efferents on the right. Inoue, Abe, et al. suggest that vagal efferents could, therefore, be the common pathway that activates the CAP. Their data add more information to ongoing efforts to understand the neural pathways involved in the CAP, which does not appear to operate via a simple reflex arc of vagal efferents forming synaptic connections with the noradrenergic postganglionic neurons in the splenic nerve, as initially thought (11). Moreover, vagal stimulation does not produce an evoked response in the splenic nerve, indicating that a vagal-vagal reflex is unlikely to be a central mechanism of CAP activation. Indeed, other data generated by Inoue, Abe, and colleagues point to more complex mechanisms, including the observation that left VNS remains protective even when the right vagal efferents are blocked during stimulation (5). This points to vagosympathetic reflex involvement or a hormonal axis.

More broadly, the studies from the Okusa lab point to the underappreciated importance of neuroimmunomodulatory mechanisms in AKI. Indeed, the AKI literature is replete with evidence that interorgan effects are important for disease development, even in simplistic models of AKI, such as the commonly employed renal artery clamp IRI model (3). This interorgan crosstalk is exemplified by the way that injury to one kidney profoundly affects the response of the other, contralateral kidney (see, for example, ref. 12). Interorgan trafficking of immune cells and dissemination of inflammatory cytokines are surely responsible for many of these effects, but the present study by Inoue, Abe et al. again reinforces the view that neural mechanisms are also likely to contribute in important ways to this phenomenon (5). Indeed, in the course of their study, the authors replicated the previous observation (13) that renal sympathetic denervation profoundly decreases injury in one model of IRI. In the setting of mul- tiorgan failure, such neural mechanisms are likely to be even more important. This is a relatively neglected aspect of AKI and one that, as this new work clearly demonstrates, deserves much more attention.

\section{Conclusions and future directions}

The effectiveness of VNS and ultrasound in mouse models is promising for further development of a practical preventative clinical strategy to reduce the incidence of AKI. Unfortunately, the ability of such an approach to treat AKI that has already started to progress is not a likely outcome, as the protective effect of neuroimmunomodulation only developed after a significant delay - VNS was effective at attenuating AKI when delivered at 24 hours but not at 2 hours prior to injury (5). Therefore, the most likely clinical use for this approach would be as a prophylactic measure in situations where the patient is at high risk of developing AKI. In favor of neuroimmunomodulation as a preventative strategy is that the treatment itself, VNS or ultrasound (the ultrasound modality used in these studies generates little heat in the target tissues), is either noninvasive or minimally invasive, and there is minimal risk from either procedure itself. Given the risk and benefit profile of this strategy, one could imagine this approach being employed widely in critical care settings to reduce the risk of the serious consequences of AKI, analogously to the provision of vaccines as low-risk preventative measures against infectious diseases. 


\section{Acknowledgments}

Research in the author's laboratory was supported by NIH grants R01 DK053194, R01 DK088934, and P30 DK079312.

Address correspondence to: Simon J. Atkinson, 755 W. Michigan Street, UL-1140, Indianapolis, Indiana 46202, USA. Phone: 317. 274.1020; E-mail: satkinso@iupui.edu.

1. Molitoris BA, et al. Design of clinical trials in AKI: a report from an NIDDK workshop. Trials of patients with sepsis and in selected hospital settings. Clin JAm Soc Nephrol. 2012;7(5):856-860.

2. Molitoris BA, Okusa MD, Palevsky PM, Kimmel PL, Star RA. Designing clinical trials in acute kidney injury. Clin JAm Soc Nephrol. 2012;7(5):842-843.
3. Zuk A, Bonventre JV. Acute kidney injury. Annu Rev Med. 2016;67:293-307.

4. Walpole H to Mann H. January 28, 1754. In: G. Dover, ed. Letters of Horace Walpole, Earl of Orford, to Sir Horace Mann, British Envoy at the Court of Tuscany. Now first published from the originals in the possession of the Earl of Waldegrave. New York, NY: George Dearborn; 1833.

5. Inoue $\mathrm{T}$, et al. Vagus nerve stimulation mediates protection from kidney ischemia-reperfusion injury through $\alpha 7 \mathrm{nAChR}^{+}$splenocytes. J Clin Invest. 2016;126(5):1939-1952.

6. Gigliotti JC, et al. Ultrasound prevents renal ischemia-reperfusion injury by stimulating the splenic cholinergic anti-inflammatory pathway. J Am Soc Nephrol. 2013;24(9):1451-1460.

7. Rosas-Ballina M, et al. Acetylcholine-synthesizing $\mathrm{T}$ cells relay neural signals in a vagus nerve circuit. Science. 2011;334(6052):98-101.

8. Gigliotti JC, et al. Ultrasound modulates the splenic neuroimmune axis in attenuating AKI. J Am Soc Nephrol. 2015;26(10):2470-2481.

9. Rosas-Ballina M, Tracey KJ. Cholinergic control of inflammation. JIntern Med. 2009;265(6):663-679.

10. Beekwilder JP, Beems T. Overview of the clinical applications of vagus nerve stimulation. JClin Neurophysiol. 2010;27(2):130-138.

11. Martelli D, Yao ST, McKinley MJ, McAllen RM. Reflex control of inflammation by sympathetic nerves, not the vagus. JPhysiol. 2014;592(7):1677-1686.

12. Basile DP, Leonard EC, Tonade D, Friedrich JL, Goenka S. Distinct effects on long-term function of injured and contralateral kidneys following unilateral renal ischemia-reperfusion. Am J Physiol Renal Physiol. 2012;302(5):F625-F635.

13. Fujii $\mathrm{T}$, et al. The role of renal sympathetic nervous system in the pathogenesis of ischemic acute renal failure. Eur J Pharmacol. 2003;481(2-3):241-248. 